\title{
A NEW ASYMMETRIC FINGERPRINTING FRAMEWORK BASED ON SECRET SHARING
}

\author{
Yan Wang, Shuwang Lü and Zhenhua Liu \\ State Key Laboratory of Information Security, \\ Chinese Academy of Science, Beijing, China * \\ ywang_cas@yahoo.com
}

\begin{abstract}
This paper presents a new type of asymmetric fingerprinting framework based on the idea of secret sharing. With the help of FIC(Fingerprint Issuing Center), we achieve an efficiency improvement in implementing asymmetric fingerprinting protocol. It avoids using secure multi-party computations. Further research directions are also suggested.
\end{abstract}

Keywords: Copyright protection, Asymmetric fingerprinting, Secret sharing

\section{INTRODUCTION}

Recent years have seen a rapid growth in the availability of multimedia content in digital form. They can be easily duplicated and redistributed. Thus there has been an increasing interest in developing copy protection or copy deterrence mechanisms. Digital watermarking has been proposed for the purpose of copyright protection for multimedia content. Digital watermark are some marks carrying the owners' information or the buyers' information, and they are embedded into the digital content without affecting the original copy's quality. Digital fingerprinting has been put forward in [Wag83]. The owner of the digital content embeds a unique digital watermark (called digital fingerprint) to each copy he sold. Thus, when the owner finds a redistributed copy, he can extract

\footnotetext{
*This paper is supported by National Key Fundamental Research Development Project(973, No.G1999035805), National High-tech Research Development Plan Youth Foundation (No.2001AA140447) and State Key Laboratory of Information Security Innovation Foundation.
}

The original version of this chapter was revised: The copyright line was incorrect. This has been corrected. The Erratum to this chapter is available at DOI: 10.1007/978-0-387-35612-9_23 
the information embedded in the redistributed copy and the information will help him trace and identify the traitor.

However, the conventional fingerprinting schemes ([BMP85], [BS95]) are in symmetric sense, i.e., both the merchant and the buyer know the copy with the buyer's fingerprint. Thus a malicious merchant could spread himself the copy sold to some buyer and then accuse that buyer of having done so. And on the other hand, a buyer whose fingerprint has been found in the redistributed copy could claim that it is the owner who wants to frame her that redistributed the copy with her fingerprint deliberately. In order to solve this problem, Pfitzmann and Schunter proposed the concept of asymmetric fingerprinting in [PS96]. The above problem is solved in the following way: After a sale, only the buyer knows the copy with the fingerprint. However, if the merchant later finds this fingerprinted copy somewhere, he could identify the buyer and obtain a proof that the buyer is a traitor. This gives both the merchant and the buyer security in disputes.

Since the concept of asymmetric fingerprinting has been put forward in [PS96], quite a few papers have discussed the constructions of asymmetric fingerprinting schemes ([PW96],[PW97_a],[PW97_b],[BM97],[Domi99] and [Cam2000]). However, those constructions rely on either secure multi-party computations ([CDG87]) or zero-knowledge proofs(ref. chap4 of [G2001]) or other cumbersome techniques which are very inefficient to realize(we will address this in section 2 again). In this paper, we present a new asymmetric fingerprinting framework based on secret sharing under some assumptions about the underlying fingerprinting scheme. Our framework is rather efficient comparing to the previous constructions.

The structure of this paper is organized as follows: Section 2 describes some related work. In section 3, we put forward an asymmetric fingerprinting framework under some assumptions about the underlying fingerprinting scheme. Some comparisons and efficiency analysis are discussed in section 4 . In section 5 , we describe a fingerprinting scheme which could be used in the framework. Some further research directions are discussed in section 6 .

\section{RELATED WORK}

In 1996 Pfitzmann put forward the concept of asymmetric fingerprinting and presented a framework in which secure multi-party computation (SMPC) is used. It is a clever idea, but not an efficient way. In [PW96], [PW97_a] and [BM97], based on the coding of [BS95], they put forward asymmetric fingerprinting schemes which can resist lager collusions. However, in their methods, secure 2-party computations are still 
be used (though the authors put forward some suggestions, they are still not efficient in practical use). In [MW98], Memon and Wong construct an asymmetric fingerprinting protocol which avoids using SMPC, but their methods only applied to some limited cases where the watermark embedding methods are some operations like $\oplus$. Moreover, using their methods, one has to calculate the total digital content to be fingerprinted using the public-key encryption and decryption operations which are known to be much less efficient than operations in symmetric cryptosystems. When the digital content is large, their method will be quite inefficient. [PW97_b] uses the similar idea of [PW96] to get asymmetric property and the fingerprinting protocol also relies on secure 2-party computation. In [Domi99], Domingo has proposed an asymmetric fingerprinting scheme based on oblivious transfer protocol. But the oblivious transfer of the image blocks is needed, which is less efficient. What's more, in the identification protocol, all buyers may have to be asked to take part in the protocol, which is a burden and unnecessary for the innocent buyers. In [Cam2000], J.Camenisch presented an anonymous fingerprinting scheme based on group signature and as a by-product, he achieved an asymmetric fingerprinting scheme with 2-party trial. However, his asymmetric scheme still based on [PW96]. In this paper, we propose a new asymmetric fingerprinting framework. It avoids using SMPC and the zero-knowledge proof primitives, which are preferred by some previous asymmetric fingerprinting protocols. The basic idea of our framework is to use secret sharing when getting the keys to decrypt the blocks of the digital content. Those blocks are pre-calculated by the merchant: Some information is embedded in the blocks and they are sent out in encrypted form. When the users get the decrypted blocks, they get their corresponding codewords (we use the basic distribution scheme in [BS95]).

\section{OUR FRAMEWORK FOR ASYMMETRIC FINGERPRINTING}

\subsection{Basic idea}

In asymmetric fingerprinting protocols, the merchant doesn't know the exact fingerprinted copy the buyer purchases. That means there must be some information the merchant doesn't know. Only if he finds the redistributed copy, can he get enough proof to prove some buyer is a traitor. The traditional way to realize asymmetric fingerprinting protocols is to use secure 2-party computations. The buyer and the merchant secretly enter their secret information and after the secure computation, the buyer gets the copy he wants, and the merchant gets his record on 
the purchase. We note that, though there may be some implementation of SMPC in this context, non of them is quite efficient. So we devise an asymmetric fingerprinting protocol without using SMPC. In our framework, we assume the codewords assigned to the buyers are binary. (But in a very similar way, it can be extended to the case of non-binary.) First, the merchant cuts his original copy into $l$ blocks (as in [BS95]), $l$ is the length of the codeword. By embedding ' 0 ' and ' 1 ' respectively into block $_{\mathbf{i}}$, he gets two versions: block $\mathrm{i}_{\mathrm{i}}^{0}$, block $\mathrm{i}_{\mathrm{i}}^{1}$ respectively. Then he uses two independent keys (keys in symmetric cryptosystem) to encrypt the two blocks respectively. He did the same thing for every block and all the keys are chosen independently. Then he sends out the $2 l$ encrypted blocks. When the buyer decrypts block $k_{i}^{0}$, the corresponding bit of her fingerprint is ' 0 ' and when decrypting block $k_{i}^{1}$, the corresponding bit of her fingerprint is ' 1 '. The basic idea of our framework is to let the merchant and the buyer share the key. Without help of the merchant, the buyer can't decrypt the blocks. And when the buyer asks the merchant for the key, the latter doesn't know the corresponding information of the buyer's codeword.

\subsection{Our framework}

Basic components:

The protocol includes 4 parties: the merchant (M), the buyer (B), the fingerprint issuing center (FIC) and the arbiter. FIC issues random binary codeword to the buyer and also issues the corresponding sub-keys of the secret sharing scheme to the buyer.

Cryptographic primitives to be used: a signature scheme and a bit commitment scheme. In our scheme, we assume that the fingerprinting scheme has reasonable collusive tolerance and tracing only relies on the merchant's initial information and his chosen random bits. i.e., without the help of buyers and without information on buyers' codeword, the merchant can deduce a possible traitor from the illegal copy and the information he stores.

There are 4 protocols: initialization, fingerprinting, identification and trial protocols.

I. Initialization: All parties generate their certificated public key and secret key pairs (Under assumed Certificated Authority) and publicize their corresponding public keys.

The merchant's pre-calculations: The merchant cuts his original copy into $l$ blocks, $l$ is the length of the codeword. By embedding ' 0 ' and ' 1 ' respectively into block ${ }_{i}$, he gets two blocks: block $_{j}^{0}$, block ${ }_{i}^{1}$. Then he 
uses two independent keys $k e y_{i}^{0}, k e y_{i}^{1}$ to encrypt the two blocks respectively. He did the same thing for every block and all the keys are chosen independently. He sends out the $2 l$ encrypted blocks. The keys used is formed as follows:

For block $\mathrm{i}_{\mathbf{i}}$, the two independent keys $k e y_{i}^{0}, k e y_{i}^{1}$ are selected from $F_{2}^{r}$, which are used as the keys for symmetric cryptosystem, e.g. DES,IDEA and AES. Then the merchant chooses another element $k e y_{i}^{m} \in F_{2}^{r}$ and computes $k e y_{i}^{f_{0}}=k e y_{i}^{0} \oplus k e y_{i}^{m}, k e y_{i}^{f_{1}}=k e y_{i}^{1} \oplus k e y_{i}^{m}$.

The merchant stores $k e y_{i}^{m}$ and sends $k e y_{i}^{f_{0}}$ and $k e y_{i}^{f_{1}}$ to FIC as subkeys.

\section{Fingerprinting:}

1. FIC randomly selects a codeword $W_{B} \in F_{2}^{l}$ for the buyer $\mathrm{B}$, signs using its secret key and then sends the codeword and signature to $B$.

2. $B$ verifies the signature using FIC's public key and decrypts the codeword using her secret key. Then B commits to $W_{B}$ bit by bit using bit commitment scheme and signs the commitments $\operatorname{Com}_{B}$ using her private key. The signature is denoted by $\operatorname{Sig}_{B}$. B sends $\left(\operatorname{Com}_{B}, \operatorname{Sig}_{B}\right)$ to $\mathrm{M}$.

3. Using B's public key $p k_{B}, \mathrm{M}$ justifies $\operatorname{Sig}_{B}$ is a valid signature on $\mathrm{Com}_{B}$ by B. Then M sends $\mathrm{Com}_{B}$ to FIC for its correctness. FIC asks the buyer $B$ to open $\operatorname{Com}_{B}$ and check whether the result equals to $W_{B}$ and if this holds, protocol continues, otherwise protocol aborts. (Note we assume FIC executes this step honestly.) And for efficiency, FIC can randomly asks the buyer to open some bits in her commitment and check whether they equal to the corresponding bits in $W_{B}$.

4. FIC sends the sub-keys to $B$ according to $W_{B}$. When the $i$-th bit of $W_{B}$ is 0 , FIC sends key $y_{i}^{f_{0}}$, otherwise $k e y_{i}^{f_{1}}(i=1$ to $l)$.

5. $B$ asks $M$ to give her another part of the keys. i.e., $M$ encrypts $k e y_{i}^{m}\left(i=1\right.$ to $l$ ) using B's public key $p k_{B}$ and sends them to B.

6. B first decrypts the keys using her private key. Upon getting $k e y_{i}^{m}$, B can compute $k e y_{i}^{0}=k e y_{i}^{f_{0}} \oplus k e y_{i}^{m}$ or $k e y_{i}^{1}=k e y_{i}^{f_{1}} \oplus k e y_{i}^{m}$. Then she could get the corresponding decrypted blocks of the digital copy, in which the embedded information coincides with her codeword.

7. $\mathrm{M}$ stores $\left(p k_{B}, \operatorname{Com}_{B}, S i g_{B}\right)$ and other information to be used in traitor tracing.

\section{III. identification:}

When $\mathrm{M}$ finds a redistributed copy, using the information he stored, $\mathrm{M}$ identifies some possible traitors and some corresponding bits which will constitute the proof. For example, he identifies $B$ and some possible bits (to say $t$ bits) of her codeword, denoted by Witness_bits $s_{B}$ and their 
corresponding locations Witness locations $s_{B}$ in the codeword. Those bits are called witness bits of $B$.

(Note that we have assumed using the underlying fingerprinting scheme, the merchant can deduce the possible traitor and some corresponding bits from the redistributed copy and the information he stores.)

IV. trial:

1. $M$ sets

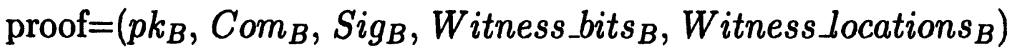
and sends the proof to the arbiter.

2. The arbiter first justifies $S_{i g}$ is a valid signature on $\operatorname{Com}_{B}$ using B's public key $p k_{B}$. Then he asks the buyer B whose public key is $p k_{B}$ to open the corresponding bit in $\operatorname{Com}_{B}$ and checks whether the corresponding bits equal to the bits in Witness_bits ${ }_{B}$. If all the bits pairs are equal, he judges the buyer $B$ is illegal. Otherwise he judges $M$ wants to frame B.

\subsection{Security analysis}

By means of security of an asymmetric fingerprinting scheme, we take the notions in [PS96]. It should be considered from three aspects: First, buyers could obtain useful data as long as nobody cheats. Second, if an illegal copy turns up, the merchant could identify some buyer who should be responsible for it. Third, buyers could be protected from cheating merchant and other buyers. We'd like to point out in practical environment, the third point is more important than the second. Because once an innocent buyer is accused of guilty, few people are willing to buy that merchant's products.

For our scheme, the first point is easy to satisfy if all the parties act according to the protocol. For the second point, we assume that under reasonable collusion size, using the underlying fingerprinting methods, the merchant could identify some traitor and several witness bits upon finding an illegal copy. The possibility the merchant could identify the traitor relies heavily on the underlying fingerprinting scheme.

For the third, we have to consider the security of the bit commitment scheme. A Bit Commitment(BC) scheme allows Alice to send something to Bob that commits her to a bit $b$ of her choice in such a way that Bob can't tell what $b$ is, but such that Alice can later prove him what $b$ originally was. The commitment obtained after the commit phase is binding if Alice cannot change the bit and it is concealing if Bob cannot obtain any information about $b$ without the help of Alice. The commitment is secure if it is binding and concealing. Here we assume 
the $\mathrm{BC}$ scheme is computationally secure. For several cryptographic realizations, see [BCC88].

We also note that in our asymmetric framework, the commitments have to be checked by FIC to prevent the buyer from concealing bit different from the corresponding bit in her codeword. Otherwise, the buyer may conceal different bits in order to deny her guilty in the trial protocol. Because the codewords assigned to the buyers are chosen randomly, thus the probability an innocent user will be framed is very small. We have the following proposition:

Proposition 1 If the codewords assigned to the buyers are chosen randomly and all the cryptographic primitives in use are secure, under the assumption that FIC executes the protocol honestly, then the probability an innocent buyer will be judged as a traitor is $1 / 2^{t}$.

Proof We take the proof under some computational assumptions (under which the cryptographic primitives we used are secure). If the merchant can frame an innocent buyer, he must get at least $t$ witness bits of that buyer. If all the parties execute the protocol honestly, it means that he can get the corresponding bits in the commitment or he guess the correct bits. But the former is contrary to the security of the bit commitment scheme, by which $M$ gets no information about the buyer's codeword. (Note that $\mathrm{M}$ also couldn't change the commitments of his will, because the buyer signs it and the arbiter could verify the signature.) So only $\mathrm{M}$ is able to guess at least $t$ witness bits correctly, can he frame an innocent buyer. This probability will not exceed $1 / 2^{t}$. And if the underlying scheme makes $t$ be large, the probability can be made negligible.

Note 1. In our protocol, we assume there are no active involvement of FIC, it can just act according to the protocol. In fact, the issuing of codewords, the issuing of sub-keys and the verification of $W_{B}$ could be programmed into a tamper-resistant hardware which can run automatically when FIC supplies it with appropriate inputs, e.g., the users' codewords. For FIC, it is of little use to take time to break the hardware. Because even if he gets access to all the sub-keys, he can't get another part of the keys to decrypt the encrypted blocks. Comparing to the case that in some framework where the merchant has to supply his original copy to certain trusted third party, our framework ensures that FIC himself can't get the copy, because he only gets sub-keys. So our framework is more feasible in practical use. 


\section{EFFICIENCY ANALYSIS}

As we have mentioned, this framework is more practical than those schemes using SMPC. In [PS96], the author also made a realization for the asymmetric fingerprinting. But the method needs the commitments to all the content of the digital copy. So the computation is prohibitive for realistic data. Other comparisons can also be found in the related work section. Here we'll show the computations and storage requirements in our framework.

Computations: Except for the computations in embedding and extracting, the merchant will do symmetric encryptions for all the subblocks two times(because there are 2 versions for each block). But for each item to be sold, the merchant need only do these operations once for all buyers. Not like the methods in [PS96] and [Domi99], whenever a buyer buys a copy, the merchant has to do the fingerprint embedding for the buyer. So our method is of much value when the copy to be fingerprinted is large, which is usually the case in practical use. Buyers need only do XOR operations and decryptions of ciphertext in symmetric cryptosystem. As to the bit commitment, the merchant, buyers and FIC need only to take some computations for the bits in the codeword, which is rather efficient comparing to making commitments to all the digital content.

Storage scale: The encrypted blocks could be publicized on the internet or to be stored by M or FIC. M has to store sub-keys for each blocks of digital content and the selling records. FIC should store two times length of the sub-keys $M$ stores. Buyers only needs to store keys temporarily and he also has to store some information used to open the commitments. E.g., if an item to be sold is divided into 512 segments and the key length is 128 bits, then the keys to be stored by $\mathrm{M}$ are $64 \mathrm{~K}$ bits. So the store scale is very reasonable.

From the above analysis, our method is advantageous when the merchant has several valuable but rather large items to sell(for example, movies, DVDs). Because each segment is encrypted and decrypted using operations in symmetric cryptosystem, our methods can achieve more efficiency than those which need public-key cryptographic operations to the items or using other cryptographic primitives(e.g. BC). But when there are many little items to be fingerprinted, we don't recommend our method, because the storage scale may be too large if we use different keys for different items. 


\section{AN ASYMMETRIC FINGERPRINTING SCHEME BASED ON REPEATED CODING}

In order to let our framework be more specific, we take a try to design a fingerprinting scheme in which $M$ could identify a traitor without the help of other parties. The idea is to use a unique pseudo sequence to control the repeated embedding of each bits in a buyer's codeword. The idea may be somewhat similar to that in [CKLS97]. But our purpose is to design a simple coding method to help the merchant identify traitors and possible witness bits.

Here we also take the same assumption as in [BS95]( the Marking Assumption): Colluding pirates can detect a specific mark location if and only if the mark differs between their copies. They cannot change an undetected mark without destroying the fingerprinted object.

The naive idea of enabling the merchant to identify one witness bit is to use repeated coding, i.e., to embed one bit many times to ensure it can be correctly retrieved. But by simple repeated embedding, if the collusive buyers have 2 versions in one mark location, they have 2 versions in all the locations where the accompanying repetition bits of that mark lie in. So they can easily change half of them to their inverse, thus the bit is hard to retrieve. Furthermore, as we have shown above, the framework requires that tracing only relies on the merchant's initial information and his chosen random bits. But in the naive idea, if the merchant doesn't know the corresponding original bits, it is rather hard to identify the correct bits. So the naive idea of simple repeated embedding is of little use. We use the idea that the merchant uses pseudo random sequences (called control sequence and denoted by $\operatorname{control}_{B}$ for buyer $B$ ) to decide half of the repetition bits will be embedded inversely. Suppose we embed one bit $d$ times, then there will be about $d / 2$ ' 0 ' and $d / 2$ ' 1 ' and they distributed evenly in the resulting repeatedly embedded bits for that particular mark. So even if the collusive buyers' mark is different in one particular location in their codewords, they can't get this information by comparing their $d$ repetition bits for the particular bit. For example(just to show the idea), if $d=10$, Two collusive buyers $B_{1}, B_{2}$ have ' 0 ' and ' 1 ' respectively in one mark. If we simply embed them $d$ times, $\mathrm{B}_{1}$ and $\mathrm{B}_{2}$ can easily detect the difference. But if we use the control sequence '11010 01011' (for $B_{1}$ ) and '01100 11010' (for $B_{2}$ ) respectively and the ' 1 ' in the control sequence indicates the corresponding bit of the original sequence will be reversed, the embedded information will be '11010 01011' (for $\mathrm{B}_{1}$ ) and '10011 00101'(for $\mathrm{B}_{2}$ ) respectively. By comparing the blocks, it is hard for them to decide 
whether the particular bit is the same. Furthermore, we can assume the embedding technique and the use of pseudo random sequence to control the inversion are kept secret from buyers. By the randomness of the control sequence, the buyer can only make some random choice in the detectable bits of the repetition bits, and by the Marking Assumption, they can't change the undetectable bits. So, for a colluder B, when using control $_{B}$ in the retrieval process to get back the original value of the repetition bits of the particular mark, the merchant will find more ' 0 ' or more ' 1 ', depending on B's mark in that particular location. However, for an innocent buyer $\mathrm{B}^{\prime}$, when using control $_{B^{\prime}}$ in the retrieval process, the merchant would see about half of the repetition bits of the particular mark are ' 0 ', and the rest are ' 1 '. Thus, using the difference between ' 0 ' and ' 1 ', not only can the merchant differentiate a colluder from an innocent buyer, but also decide the original mark of the colluder with high probability by taking reasonable threshold value.

Most parts of the protocol using the above coding method are the same with those in the framework in section 3.2. But in order to let the merchant embed the mark inversely, the merchant will make precalculations for $L=l d$ blocks, where $d$ is the repetition times of a single bit. The difference is: For block,$(i$ is from 1 to $L$,$) the merchant$ selects four independent keys $k e y_{i}^{0}, k e y_{i}^{1}, k e y_{-} r_{i}^{0}, k e y_{-} r_{i}^{1} \in F_{2}^{r}$ and he chooses another two element $k e y_{i}^{m}, k e y_{-} r_{i}^{m} \in F_{2}^{r}$ and computes $k e y_{i}^{f_{0}}=$ $k e y_{i}^{0} \oplus k e y_{i}^{m}, k e y_{i}^{f_{1}}=k e y_{i}^{1} \oplus k e y_{i}^{m}$ and key_r $r_{i}^{f_{0}}=k e y_{-} r_{i}^{0} \oplus k e y_{-} r_{i}^{m}$, $k e y_{-} r_{i}^{f_{1}}=k e y_{-} r_{i}^{1} \oplus k e y_{-} r_{i}^{m} . \quad k e y_{i}^{0}, k e y \_r_{i}^{1}$ are used to encrypted block $\mathrm{i}_{\mathrm{i}}^{0}$, $k e y_{i}^{1}, k e y_{-} r_{i}^{0}$ are used to encrypted block ${ }_{\mathrm{i}}^{1}$. (Note that key_r $r_{i}^{0}, k e y_{-} r_{i}^{1}$ and key $r_{i}^{m}$ are used for inversely embedding.) For one block, $\mathrm{M}$ gets 4 encrypted blocks. So $M$ has to pre-calculate $4 L$ encrypted blocks.

$\mathrm{M}$ stores $k e y_{i}^{m}$ and key_r $r_{i}^{m}$ and sends $K E Y 0=\left\{k e y_{i}^{0}, k e y_{-} r_{i}^{0}\right\}$ and $K E Y 1=\left\{k e y_{i}^{1}, k e y \_r_{i}^{1}\right\}$ to FIC as sub-keys. In the fingerprinting protocol, FIC will send the sub-keys to $\mathrm{B}$ according to $W_{B}^{\prime}=W_{B, 1} W_{B, 1} \ldots W_{B, 1}$ $W_{B, 2} W_{B, 2} \ldots W_{B, 2} \ldots \ldots W_{B, l} W_{B, l} \ldots W_{B, l}$ (every $W_{B, j}$ appears $d$ times, $\left|W_{B}^{\prime}\right|$ $=d l=L)$. When the $i$-th bit of $W_{B}^{\prime},(i=1$ to $L)$ is 0 , FIC gives $K E Y 0$, otherwise gives $K E Y 1$. M will send his sub-keys according the pseudo sequence for $\mathrm{B}$. If the corresponding bits is 0 , he sends $k e y_{i}^{m}$ otherwise $k e y_{-} r_{i}^{m}$. Thus he can implement the reversely embedding of some bits while not knowing any information on B's codeword.

\section{CONCLUSIONS}

In this paper we present a new idea for asymmetric fingerprinting based on secret sharing. We also describe an idea for the underlying fingerprinting scheme. When the digital data to be fingerprinted is large, 
our framework is quite efficient. Our future research will include to devise fingerprinting methods satisfying the assumption for the underlying fingerprinting scheme in the framework. In addition, though the trust requirement for FIC is lower than that for a real TTP, it is still necessary to make some trust assumptions on FIC in our framework. Our future research will pay more considerations on the role of FIC and how to decrease the trust requirement on FIC. What's more, deep investigations on other secret sharing scheme like verifiable secret sharing may give us good suggestions for the constructions of asymmetric fingerprinting protocol.

\section{References}

[BCC88] G. Brassard, D. Chaum and C. Crepeau, Minimum disclosure proofs of knowledge,Journal of Computer and System Sciences(JCSS), 37(2), pp.156189,1988 .

[BMP85] G. R. Blakley, C. Meadows and G. B. Purdy, Fingerprinting Long For-giving Messages, Proceedings of Crypto'85, Springer-Verlag, pp.180-189, 1985.

[BM97] I. Biehl and B. Meyer,Protocols for Collusion-Secure Asymmetric Fingerprinting, Proc. 14th Annual Symposium on Theoretical Aspect of Computer Science, Springer-Verlag, pp.399-412,1997.

[BS95] D. Boneh and J. Shaw, Collusion-Secure Fingerprinting for Digital Data, Advances in Cryptology: Proceedings of Crypto'95, Springer-Verlag, pp.452-465, 1995.

[BS98] D. Boneh and J.Shaw, Collusion-Secure Fingerprinting for Digital Data, IEEE Trans.Inform.Theory, vol IT-44, pp.1897-1905, Sep.1998.

[CAM2000] J. Camenisch, Efficient Anonymous Fingerprinting with Group Signatures, In Advances in Cryptology -Asiacrypt 2000. volume 1976 of LNCS, Springer Verlag, pp.415-428, 2000.

[CDG87] David Chaum, Ivan B. Damgard and Jeroen van de Graaf, Multiparty Computations Ensuring Privacy of Each Party's Input and Correctness of the Result, Advances in Cryptology - CRYPTO'87 Proceedings, Springer-Verlag, pp.87-119, 1988.

[CKLS97] I.Cox, J.Killian, T.Leighton and T.Shamoon, Secure spread spectrum watermarking for multimedia, IEEE Transactions on Image Processing, vol. 6, no.12, pp.1673-1687, December 1997.

[Domi99] J. Domingo-Ferrer,Anonymous fingerprinting based on committed oblivious transfer,in Public Key Cryptography'99 (Lecture Notes in Computer Science 1560), eds. H. Imai and Y. Zheng, Berlin: Springer-Verlag, pp.43-52, 1999.

[G2001] O.Goldreich,Foundations of Cryptography:Volume 1-Basic tools, Cambridge University Press, 2001

[MW98] Nasir Memon and Ping Wah Wong,A Buyer-Seller Watermarking Protocol, IEEE Signal Processing Society 1998 Workshop on Multimedia Signal Processing, Los Angeles, California, USA. Electronic Proceedings, 1998.

[PS96] B. Pfitzmann and M. Schunter,Asymmetric Fingerprinting, Advances in Cryptology - EUROCRYPT'96, Springer-Verlag, pp.85-95, 1996. 
[PW96] B. Pfitzmann and M. Waidner, Asymmetric Fingerprinting for Larger Collusions, IBM Research Report RZ 2857(\# 90805)08/19/96, IBM Research Division, Zurich, 1996.

[PW97_a] B. Pfitzmann and M. Waidner, Asymmetric Fingerprinting for Larger Collusions, 4th ACM Conference on Computer and Communications Security, Zürich, pp.151-160, 1997.

[PW97_b] B. Pfitzmann and M. Waidner, Anonymous Fingerprinting, Advances in Cryptology - EUROCRYPT'97, Springer-Verlag, pp.88-102, 1997.

[Wag83] N. Wagner,Fingerprinting,Proceedings of the 1983 Symposium on Security and Privacy, pp.18-22, 1983. 